\title{
COPD - Historischer Überblick, aktuelles Management, Forschungsperspektiven
}

\author{
COPD - A Historical Review, Current Management and Research Perspectives
}

Autoren

Institute
C. Vogelmeier ${ }^{1}$, H. Worth ${ }^{2}$

Direktor der Klinik für Innere Medizin mit Schwerpunkt Pneumologie, Universitätsklinikum Gießen und Marburg

${ }^{2}$ Medizinische Klinik I, Klinikum Fürth eingereicht 9.7.2010

akzeptiert nach Revision 23. 7.2010

\section{Bibliografie}

DOI http://dx.doi.org/

10.1055/s-0030-1255678

Pneumologie 2010; 64: 550-554 @ Georg Thieme

Verlag KG Stuttgart · New York ISSN 0934-8387

Korrespondenzadresse

Prof. Dr. H. Worth

Medizinische Klinik I

Klinikum Fürth

Jakob-Henle-Str. 1

90766 Fürth

med1@klinikum-fuerth.de

\section{Zusammenfassung}

$\nabla$

Der Begriff COPD, der chronisch obstruktive Bronchitis und Lungenemphysem zusammenfasst, wurde erst 1964 geprägt. Meilensteine für das Verständnis der Erkrankung waren die Aufdeckung des Zusammenhangs zwischen Tabakkonsum und Entwicklung einer chronisch obstruktiven Bronchitis sowie die Entwicklung eines Lungenemphysems als Folge einer gestörten Homöostase zwischen Proteinasen und Antiproteinasen. Die funktionsanalytische Diagnostik der COPD wurde insbesondere in den 60er-Jahren, die Quantifizierung und Lokalisation des Lungenemphysems mittels HR-CT vor allem in den 80er-Jahren entwickelt. Das Management der Volkskrankheit COPD umfasst präventive Maßnahmen (Verzicht auf Tabakrauchen, Influenzaschutzimpfung, Arbeitsplatzhygiene), medikamentöse Therapieoptionen (Bronchodilatatoren, Kortikosteroide), nicht medikamentöse Therapieoptionen (körperliches Training, Patientenschulung, physiotherapeutische Atemtherapie) sowie apparative und operative Behandlungsoptionen (Bullektomie, Lungenvolumenreduktion, Lungentransplantation). Besonders wichtig sind die Prävention und die rasche Beherrschung von Exazerbationen, die den Krankheitsverlauf ungünstig beeinflussen. Das optimierte Management der COPD muss die Auswirkungen der Erkrankung auf andere Organsysteme berücksichtigen. Die Forschungsperspektive liegt im Aufbau großer internationaler und nationaler Netzwerke, mit denen auch die Genetik der Erkrankung analysiert werden kann.

\section{Historische Entwicklung}

Der Begriff COPD (chronic obstructive pulmonary disease) ist erst in den 60er-Jahren in den USA geprägt worden [1-3]. Weit in die Medizingeschichte zurückreichend finden sich Darstellun-

\section{Abstract \\ $\nabla$}

The term COPD which comprises chronic obstructive bronchitis and emphysema, was first defined in 1964. Key milestones to the understanding of the etiopathology of COPD were the discovery of the association between tobacco consumption and the development of chronic bronchitis as well as the discovery of the role of a protease-antiprotease imbalance leading to emphysema. The assessment of functional impairement of patients with COPD was established in the 1960s and the quantification and localisation was predominantly explored in the 1980s. The management of COPD comprises preventative measures (e.g. smoking cessation, vaccination against influenza, reduction of occupation hazards), medical therapies (bronchodilators, corticosteroids), non-pharmacological therapies (exercise training, patient education, physiotherapy) as well as surgical options. The prevention and efficiency management of exacerbations are of particular importance. Optimal management of COPD has to take impact of the disease on other organs into account. The future of COPD-related research lies in the development of international and national networks to facilitate the analysis of genetic factors on the pathology of COPD. 


\section{Entwicklung der Untersuchungsmethoden}

$\nabla$

Laennec [6], der 1815 das Stethoskop erfand, korrelierte strukturelle Veränderungen des Emphysems mit klinischen Befunden und beschrieb auch die COPD-Exazerbation in Folge einer Verschlechterung durch einen „, akuten Katarrh“ [6].

In den 30er-Jahren lagen bereits komplette Beschreibungen der radiologischen Befunde bei schwerem Emphysem vor. Die computertomografische Lokalisation und Quantifizierung des Emphysems anhand der Lungendichte mittels HR-CT wurde in den 80er-Jahren entwickelt und ist heute das präziseste diagnostische Verfahren zur Charakterisierung des Emphysems.

\section{Lungenfunktionsanalytische Diagnostik bei COPD \\ $\nabla$}

Die funktionsanalytische Diagnostik der COPD eröffnete die Möglichkeit, die funktionelle Beeinträchtigung durch die chronisch obstruktive Bronchitis und das Lungenemphysem zu messen. Die Aufzeichnung spirometrischer Volumen-Zeit-Kurven führte zur Darstellung niedriger Atemzugvolumina und einer verlängerten Exspirationsdauer bei Patienten mit Emphysem [7]. Christie [8] beschrieb die mechanischen Eigenschaften der Emphysemlunge, die durch einen Anstieg des Atemwiderstandes und eine verminderte elastische Rückstellkraft gekennzeichnet sind. Tiffeneau führte 1947 das nach ihm benannte Manöver der forcierten Exspiration in die Diagnostik obstruktiver Atemwegserkrankungen ein [9].

In den 60er-Jahren stellte Bates [10] eine Einschränkung der Diffusionskapazität der Lunge bei Patienten mit Emphysem fest, die Arbeitsgruppe von T. Macklem, J. Mead sowie J. C. Hogg [11] lokalisiert den erhöhten Atemwegswiderstand bei COPD-Patienten vor allem in den peripheren Atemwegen. In Deutschland wurde die Bodyplethysmografie zur Diagnostik und Differenzialdiagnostik der obstruktiven Atemwegserkrankungen etabliert $[12,13]$, die eine Differenzierung nach Schweregrad und Lokalisation der Obstruktion ermöglichte sowie über die Messung des intrathorakalen Gasvolumens auch eine Abschätzung der Lungenüberblähung.

\section{Entwicklung der Vorstellungen zur Pathogenese \\ $\nabla$}

Zwei Theorien zur Pathogenese beherrschten die Literatur des 19. und des frühen 20. Jahrhunderts - die mechanische und die ischämische oder atrophische. Von manchen Autoren wurde angenommen, dass eine exzessive inspiratorische Kraft über Dehnung von Lungengewebe zu einem Schaden führt. Allerdings war die dominierende Vorstellung, dass eine exspiratorisch wirksame Obstruktion ursächlich ist. Laennec [6] erwog, dass das Emphysem die Folge sei von Luft, die während der Exspiration gefesselt wurde. Bronchiolen würden als Folge von Obstruktion oder Mukosaverdickung wie ein Ventil wirken.

Die ischämische oder atrophische Theorie versuchte, das Verschwinden der Lungenkapillaren und die Entwicklung der Fenestrae zu erklären, durch Thrombosen und Gefäßobliteration als Folge eines erhöhten Alveolardrucks.

Laurell und Eriksson [14] berichteten über den Nachweis eines schweren Mangels an Serum-Alpha-1-Globulin bei 5 Individuen mit unterschiedlichen klinischen Präsentationen. Drei davon hatten Atemwegserkrankungen mit chronischer Bronchitis, Bron- chiektasen und Emphysem. Das fehlende Protein wurde Alpha1-Antitrypsin genannt.

Zeitgleich wurden von Gross [15] Tierexperimente durchgeführt, bei denen das elastolytische Enzym Papain in die Lungen von Ratten eingebracht wurde. Diese Maßnahme führte zur Ausbildung eines Emphysems.

Aus diesen Befunden - vom Menschen und aus den Tiermodellen - entwickelte sich die Vorstellung, dass das Emphysem als Folge einer gestörten Homöostase zwischen Proteasen und Antiproteasen in der Lunge entsteht.

\section{Erste Untersuchungen zur Epidemiologie und dem klinischen Verlauf \\ $\nabla$}

1961 wurde die sogenannte holländische Hypothese von der Arbeitsgruppe von Orie [16] aufgestellt, die Asthma, chronische Bronchitis und Emphysem auf Umweltfaktoren wie Rauchen und Allergien sowie eine genetische Disposition zurückführte. Mitchell und Filley [1-3] schufen 1964 den Begriff „COPD“, zunächst sicher nur in der Intention, Asthma bronchiale einerseits und ein Gemisch weiterer Atemwegserkrankungen andererseits voneinander abzugrenzen, obgleich sowohl Asthma als auch COPD eine Bronchialobstruktion aufweisen.

Von großer Bedeutung für die weitere Entwicklung war auch die Arbeit von Fletcher [17] et al. aus dem Jahr 1977, die einen Abfall des $\mathrm{FEV}_{1}$ in einer Untergruppe von Rauchern fanden, der wesentlich größer war als beim Durchschnitt der Bevölkerung. Sie zeigten, dass Raucher, die mit dem Rauchen aufhörten, einen normalen altersbedingten Abfall des $\mathrm{FEV}_{1}$ aufweisen. Daraus entwickelte sich zum einen die Vorstellung der Suszeptibilität gegenüber den Folgen des Rauchens für die Lunge, ohne dass die Ursachen dieser Empfänglichkeit definiert werden konnten.

Zum anderen wurde die Erwartung abgeleitet, dass die Beendigung des Zigarettenrauchens einen modifizierenden Einfluss auf den Verlauf der Lungenfunktion hat. Letzteres wurde später durch die Ergebnisse der Lung-Health-Studie aus Nordamerika bestätigt [18].

\section{Gegenwärtiges Management der COPD \\ $\nabla$}

Die COPD, die die chronisch obstruktive Bronchitis und das Lungenemphysem umfasst, ist durch eine nicht vollständig reversible Atemwegsobstruktion gekennzeichnet. Weltweit ist die COPD gegenwärtig die vierthäufigste Todesursache. Nach einer aktuellen Morbiditätsprognose häufiger Erkrankungen in Deutschland wird im Jahre 2007 von 6,4 Millionen an COPD Erkrankten ausgegangen. Für das Jahr 2030 wird eine Zunahme von $23 \%$ erwartet [19].

\section{Diagnostik}

Charakteristische Symptome sind Dyspnoe unter Belastung sowie chronischer Husten und Auswurf. Wichtigster Risikofaktor ist das Zigarettenrauchen. Wesentlich für diese häufig unterbzw. fehldiagnostizierte Erkrankung ist es, bei der Angabe von chronischem Husten mit und ohne Auswurf sowie Vorliegen von Risikofaktoren (inhalativer Tabakkonsum, berufsbedingte Stäube, allgemeine Luftverschmutzung, häufige Atemwegsinfektio- 


\begin{tabular}{|c|c|c|c|}
\hline Prävention & $\begin{array}{l}\text { Medikamentöse } \\
\text { Behandlung }\end{array}$ & $\begin{array}{l}\text { Nicht medikamentöse } \\
\text { Behandlung }\end{array}$ & $\begin{array}{l}\text { Apparative/operative } \\
\text { Behandlung }\end{array}$ \\
\hline $\begin{array}{l}\text { Raucherentwöhung } \\
\text { Schutzimpfungen } \\
\text { Arbeitsplatzhygiene }\end{array}$ & $\begin{array}{l}\text { Beta-2-Sympathomimetika } \\
\text { Anticholinergika } \\
\text { Theophyllin } \\
\text { Glukokortikoide } \\
\text { Mukopharmaka } \\
\text { Antibiotika }\end{array}$ & $\begin{array}{l}\text { körperliches Training } \\
\text { Patientenschulung } \\
\text { Physiotherapie } \\
\text { Ernährungsberatung }\end{array}$ & $\begin{array}{l}\text { Langzeit- } \mathrm{O}_{2} \text {-Therapie } \\
\text { nicht invasive Beatmung } \\
\text { Emphysemchirurgie }\end{array}$ \\
\hline
\end{tabular}

Tab. 1 Therapieoptionen bei COPD [20].

\begin{tabular}{|c|c|c|c|c|c|}
\hline Charakteristika & $\begin{array}{l}\text { FEV } / \mathrm{VC}<70 \% \\
\text { FEV } 1 \geq 80 \% \\
\text { mit/ohne } \\
\text { Symptomatik }\end{array}$ & $\begin{array}{l}>\mathrm{FEV}_{1} / \mathrm{VC}<70 \% \\
50 \%>\mathrm{FEV}_{1}<80 \% \\
\text { - mit/ohne } \\
\text { Symptomatik }\end{array}$ & $\begin{array}{l}\text { - } \mathrm{FEV}_{1} / \mathrm{VC}<70 \% \\
\text { > } 30 \%>\mathrm{FEV}_{1}<50 \% \\
\text { > mit/ohne } \\
\text { Symptomatik }\end{array}$ & $\begin{array}{l}\mathrm{FEV}_{1} / \mathrm{VC}<70 \% \\
\text { FEV }_{1}<30 \% \text { oder } \\
\text { respir. Insuffizienz/ } \\
\text { Zeichen der } \\
\text { Rechtsherz- } \\
\text { insuffizienz }\end{array}$ & $\begin{array}{l}\text { Abb. } 1 \text { Schweregradorientierte Therapie der } \\
\text { COPD [20]. FEV } \mathrm{V}_{1} \text { : nach Bronchodilatation bestimm- } \\
\text { te Einsekundenkapazität, VC: inspiratorische Vital- } \\
\text { kapazität. }\end{array}$ \\
\hline & \multicolumn{4}{|c|}{$\begin{array}{l}\text { Vermeidung von Risikofaktoren; Grippe- und Pneumokokken-Schutzimpfung } \\
\text { Zusätzlich bei Bedarf kurzwirksamer Bronchodilatator }\end{array}$} & \\
\hline & & $\begin{array}{l}\text { Zusätzlich D } \\
\text { langwirksaı }\end{array}$ & $\begin{array}{l}\text { herapie mit einen } \\
\text { ronchodilatatore }\end{array}$ & $\begin{array}{l}\text { oder mehreren } \\
\text { Rehabilitation }\end{array}$ & \\
\hline & & & $\begin{array}{l}\text { Zusätzlich inhal } \\
\text { wiederkehrende }\end{array}$ & $\begin{array}{l}\text { tive Steroide bei } \\
\text { h Exazerbationen }\end{array}$ & \\
\hline & & & & $\begin{array}{c}\text { Zusätzlich } \\
\text { Langzeitsauerstoff- } \\
\text { therapie bei respir. } \\
\text { Insuffizienz; Prüfen } \\
\text { ob eine chirurgische } \\
\text { Behandlung } \\
\text { angezeigt ist }\end{array}$ & \\
\hline
\end{tabular}

nen in der Kindheit) die Verdachtsdiagnose einer COPD zu stellen und abzuklären.

Als Kriterien einer COPD werden eine Obstruktion mit einem Wert des Quotienten $\mathrm{FEV}_{1} / \mathrm{VC}<70 \%$ und einer Reversibilität der $\mathrm{FEV}_{1}$ von $<15 \%$ und $<200 \mathrm{ml}$ gegenüber dem Ausgangswert nach Inhalation eines kurzwirksamen Beta-2-Sympathomimetikums bzw. Anticholinergikums angegeben [20]. Differenzialdiagnostisch ist die COPD insbesondere gegenüber dem Asthma bronchiale abzugrenzen, das in der Regel eine höhere Reversibilität der Obstruktion, eine ausgeprägtere bronchiale Hyperreaktivität, einen frühzeitigeren Krankheitsbeginn und eine höhere Variabilität der Symptome aufweist.

\section{Therapie \\ $\nabla$}

Das Management der COPD ( $\bullet$ Tab. 1) umfasst die Prävention, die medikamentöse Therapie sowie nicht medikamentöse, apparative und operative Behandlungsoptionen.

Einsatz und Auswahl der Therapieoptionen ( $\bullet$ Tab. 1) haben die Kooperationsbereitschaft des Patienten und seine Komorbiditäten zu berücksichtigen.

\section{Prävention}

$\nabla$

Wichtigste präventive Maßnahme ist der Verzicht auf das Tabakrauchen. Multimortale Raucherentwöhnungsprogramme mit Verhaltenstherapie, sozialer Unterstützung, einer Pharmakotherapie zur Behandlung der Nikotinabhängigkeit (Nikotinkaugummi, Nikotinspray, Nikotinpflaster) sowie mit suchthemmenden Medikamenten (Vareniclin, Buproprion) können die Erfolgs- quoten der Entwöhnung deutlich steigern. Bei rückfälligen Rauchern sollte ein erneuter Entwöhnungsversuch durchgeführt werden.

\section{Schutzimpfungen}

Bei allen Patienten mit COPD sollte im Herbst die Influenzaschutzimpfung mit der jeweils aktuellen Vaxine erfolgen [20]. Eine einmalige Pneumokokkenschutzimpfung wird für alle COPD-Patienten empfohlen.

\section{Langzeitbehandlung}

Die Langzeitbehandlung der stabilen COPD ist durch eine schrittweise Erweiterung der Behandlungsoptionen in Abhängigkeit vom Schweregrad der Erkrankung ( $\bullet$ Abb. 1) gekennzeichnet. Im Gegensatz zur Raucherentwöhnung lässt sich mit keinem der vorhandenen, medikamentösen Therapieansätze, mit Ausnahme der Langzeit- $\mathrm{O}_{2}$-Therapie, die Prognose bessern [20]. Die Pharmakotherapie ermöglicht eine Linderung der Beschwerden, eine Besserung von körperlicher Leistungsfähigkeit und Lebensqualität sowie eine Verminderung der Exazerbationsfrequenz. Neuere Untersuchungen an Patienten mit mittelgradiger COPD zeigen, dass für diese Patienten unter Inhalation von Tiotropium der jährliche Funktionsverlust geringer ist als unter Placebo. Dies spricht für einen frühzeitigen Beginn einer Dauerbehandlung mit Bronchodilatatoren bereits im Stadium II [21].

Eine Dauerbehandlung mit inhalativen Glukokortikoiden sollte bei Patienten mit einer postbronchodilatorischen $\mathrm{FEV}_{1}<50 \%$ Soll und mindestens einer den Einsatz von systemischen Gluko- 


\begin{tabular}{|c|c|c|}
\hline Schweregrad & Kennzeichen & Therapie \\
\hline schwergradig & $\begin{array}{l}\text { Hyper- }(>30 / \mathrm{min}) \text { od. } \\
\text { Hypoventilation }(<12 / \mathrm{min}) \\
\text { Bewusstseinseintrübung } \\
\text { Tachykardie } \\
\text { Zyanose (neu/progred.) } \\
\text { Ödeme }\end{array}$ & $\begin{array}{l}\text { zusätzlich: } \\
\mathrm{O}_{2} \text { bei Hypoxämie } \\
\text { nicht invasive Beatmung bei Hyperkapnie und } \\
\text { respiratorischer Azidose } \\
(\mathrm{pH}<7,35)\end{array}$ \\
\hline mittelgradig & $\begin{array}{l}\text { Atemnot } \uparrow / \text { Husten } \uparrow \\
+ \text { Verschlechterung der Lungenfunktion } \\
\text { bedeutsame Komorbidität }\end{array}$ & $\begin{array}{l}\text { zusätzlich: } \\
\text { system. Kortikosteroide } \\
\text { bei fehlender Besserung evtl. Theophyllin }\end{array}$ \\
\hline leichtgradig & $\begin{array}{l}\text { leichte subj. Verschlechterung } \\
\pm \text { Verschlechterung der Lungenfunktion }\end{array}$ & $\begin{array}{l}\text { Intensivierung der Ther. mit ACH und/oder } \\
\text { B2S-Anticholinergika u./o. Beta-2-Sympatho- } \\
\text { mimetika }\end{array}$ \\
\hline alle Schweregrade & & $\begin{array}{l}\text { Nikotinverzicht, Antibiotika bei purulentem } \\
\text { Sputum, Ther. der Komorbidität }\end{array}$ \\
\hline
\end{tabular}

Tab. 2 Schweregradorientierte Therapie der COPDExazerbation [20]. kortikoiden und/oder Antibiotika notwendig machenden COPDExazerbation im letzten Jahr zum Einsatz kommen [20].

Neben Bronchodilatatoren (kurz- und langwirksame Anticholinergika bzw. Beta-2-Sympathomimetika Theophyllin) und inhalativen Glukokortikoiden werden mit geringerer Evidenz auf individueller Basis Mukopharmaka eingesetzt.

\section{Nicht medikamentöse Therapie}

$\nabla$

Körperliches Training führt bei Patienten mit COPD zu einer Linderung der Dyspnoe, einer Zunahme der Belastbarkeit und einer Steigerung der Lebensqualität.

Positive Effekte werden insbesondere bei Trainingsprogrammen mit 3-5 Trainingseinheiten pro Woche erzielt. Wesentlich für die Aufrechterhaltung der Trainingseffekte ist die Etablierung einer wohnortnahen Bewegungstherapie, auch unter Nutzung ambulanter Lungensportgruppen [20].

Die Patientenschulung ist ein wesentliches Therapieelement für COPD-Patienten aller Schweregrade, da sie zu einer Steigerung der Effizienz des Managements in Folge des Monitorings der Symptome durch den Patienten und durch die schweregradadaptierte Selbstmedikation führt.

Die Langzeittherapie wird unterstützt durch spezifische Maßnahmen der physiotherapeutischen Atemtherapie und bei an Gewicht abnehmenden Patienten auch durch eine geeignete Ernährungstherapie [20].

Indikationen zur ambulanten oder stationären Rehabilitation sind gegeben, wenn trotz adäquater Krankenhausbehandlung körperliche oder psychosoziale Krankheitsfolgen persistieren, welche alltagsrelevante Aktivitäten und die Teilhabe am normalen privaten, öffentlichen oder beruflichen Leben behindern [20]. Bei Patienten mit chronischer Hypoxämie führt die Langzeitsauerstofftherapie bei Anwendung über 16-24 Stunden pro Tag zu einer Besserung der Prognose [20].

Bei Patienten mit Hyperkapnie, bei denen die Atemmuskulatur durch die erhöhte Atemarbeit vermehrt beansprucht ist, kann die Indikation zur intermittierenden nicht invasiven Beatmung dann gestellt werden, wenn alle konservativen Behandlungsmöglichkeiten ausgeschöpft sind und der Patient weiterhin hyperkapnisch ist [20].

Die Indikationen zu operative Therapieverfahren wie Bullektomie, Lungenvolumenreduktion und Lungentransplantation sind der aktuellen deutschen COPD-Leitlinie [20] zu entnehmen.

\section{Management der Exazerbationen}

Unter einer Exazerbation der COPD wird eine akute Verschlechterung mit Zunahme von Symptomen (Atemnot, Husten, Auswurf, thorakale Beklemmung, selten Fieber) verstanden, die über die täglichen Schwankungen hinausgeht und eine Änderung der Medikation erforderlich macht ( Tab. 2).

Häufigste Ursachen der Exazerbationen sind virale und/oder bakterielle Atemwegsinfekte. Die schweregradorientierte Behandlung der Exazerbationen sieht eine Intensivierung der Therapie mit Bronchodilatatoren, den zeitlich auf maximal 14 Tage beschränkten Einsatz systemischer Glukokortikosteroide sowie die Gabe von Antibiotika bei bakteriellen Bronchialinfekten vor (๑ Tab. 2).

Bei respiratorischer Insuffizienz sollte Sauerstoff unter Kontrolle der arteriellen Blutgase appliziert werden, bei zusätzlich bestehender Hyperkapnie und respiratorischer Azidose hat sich die nicht invasive Beatmung mit positivem Druck bewährt.

Die Prävention von Exazerbationen kann durch die Vermeidung inhalativer Noxen, die Schutzimpfung gegenüber Influenza, durch inhalative Anticholinergika, inhalative Glukokortikoide, inhalative langwirksame Beta-2-Sympathomimetika, Cineol sowie die Patientenschulung erreicht werden.

Das optimierte Management der COPD muss den Patienten und die Auswirkungen seiner COPD auf andere Organsysteme mit Beeinträchtigung der Psyche (Depression), des Herz-Kreislauf-Systems, der Muskulatur, des Ernährungszustandes und des Skelettsystems berücksichtigen. Zur Optimierung des Behandlungserfolgs ist ein Netzwerk kompetenter Betreuer anzustreben. Hierzu gehören Hausarzt wie Facharzt, Akutkrankenhaus und Rehabilitationsklinik, Physiotherapeut und Übungsleiter ambulanter Lungensportgruppen sowie gelegentlich auch Sozialpädagogen und Psychologen.

\section{Zukunft der COPD-Forschung}

Die Zukunft der COPD-Forschung wird aus Sicht der Autoren durch zwei wesentliche Aspekte bestimmt werden, die auch noch miteinander verknüpft sind: Da ist die Notwendigkeit der Entwicklung von Medikamenten, die den Krankheitsverlauf elementar modifizieren. Derartige Entwicklungen setzen voraus, dass die Pathogenese der Erkrankung besser verstanden wird. Dies kann nur über die systematische Analyse von biologischen Materialien gelingen, die von genau charakterisierten Patientenkollektiven gewonnen werden, nachdem nach wie vor keine Tier- 
modelle existieren, die die COPD in ihrer ganzen Komplexität abbilden können.

Dazu bedarf es des Aufbaus von großen Netzwerken: Im Jahr 2008 wurde auf Ausschreibung des Bundesministeriums für Bildung und Forschung das Deutsche Asthma- und COPD-Netzwerk gegründet. Das zentrale Projekt in der ersten Förderperiode ist die Entwicklung einer Kohorte mit 3000 COPD-Patienten aller Schweregrade, die longitudinal nachverfolgt werden sollen. Die Kohorte soll die Frage beantworten helfen, inwieweit Erkrankungen außerhalb der Lunge, die bei der COPD häufig beobachtet werden (z.B. Kachexie, Gewichtsverlust, Muskelatrophie, Muskelschwäche, Osteoporose, kardiovaskuläre Erkrankungen, Tumoren, metabolisches Syndrom, Depression und Angstattacken) durch eine systemische Entzündung ausgelöst werden [22]. Entscheidende Voraussetzung für das Gelingen solcher Initiativen ist der Aufbau entsprechender Banken für Biomaterialien und Bilder. Dazu haben sich 7 Universitäten, das Helmholtz-Zentrum München, das Max-Planck-Institut in Bad Nauheim und 20 klinische Studienzentren, die über Deutschland verteilt sind, zusammengefunden.

Vor diesem Hintergrund ist auch die kürzliche Entscheidung der Bundesregierung, ein deutsches Zentrum für Lungenforschung auszuschreiben, von enormer Bedeutung. Damit besteht die einmalige Option, eine dauerhafte, hoch leistungsfähige Infrastruktur zu schaffen, die die Bearbeitung großer Fragestellungen in einer Weise ermöglichen wird, die international konkurrenzfähig ist.

Wie wichtig das für die weitere Entwicklung der COPD-Forschung in Deutschland sein wird, sei an folgendem Beispiel erläutert. Vor kurzem fand ein erstes Treffen aller weltweit bekannten COPD-Netzwerke in Boston statt. Dabei kamen insgesamt 30 Gruppen zusammen. Ziel des Treffens war es, die Erforschung der Genetik der COPD mittels genome wide association screens (GWAS) voranzubringen. Diese Analysen, die nur auf der Basis von großen Patientenkohorten und Vergleichskollektiven möglich sind, sind eine der Voraussetzungen dafür, neue Erkenntnisse zur Pathogenese der COPD zu gewinnen. Um es in einem Satz zusammenzufassen: Die COPD-Forschung der Zukunft wird in hohem Maße international sein. Um in diesem Konzert eine wesentliche Rolle spielen zu können, bedarf es einer leistungsfähigen nationalen Infrastruktur. Angesichts der oben aufgeführten Initiativen haben wir eine realistische Chance, derartige Strukturen zu entwickeln.

\section{Interessenkonflikte}

$\nabla$

Die Autoren geben an, dass kein Interessenkonflikt besteht.

\section{Literatur}

1 Mitchell RS, Ryan SF, Petty TL, Filley GF. Significance of morphologic chronic hyperplastic bronchitis. Am Rev Respir Dis 1966; 93: 720

2 Filley GF. Emphysema and chronic bronchitis: clinical manifestations and their physiologie significance. Med Cin North Am 1967; 51: 283 292

3 Filley GF, Dart GA, Mitchell RS. Emphysema and chronic bronchitis:clinical manifestations and their physiological significance Aspen. Emphysema Conf 1968; 9: 339-349

4 Snider GL. Emphysema: the first two centuries - and beyond. Am Rev Respir Dis 1992; 146: 1334- 1344 und 1992; 146: 1615-1622

5 Baillie $M$. The morbid anatomy of some of the most important parts of the human body. 2nd American ed. From the 3rd London ed., corrected. Brattleborough, VT: W Fessenden, 1808: 52-53

6 Laennec RTH. A treatise on the diseases of the chest and on mediate auscultation. John Forbes trans. From the 4th London ed. Philadelphia: Desilver, Thomas \& Co., 1835: 135-163

7 Salter HH. Lectures on dyspnea. Lancet 1866; 84: 153-162

8 Christie RV. The elastic properties of the emphysemateous lung and their clinical significance. J Clin Invest 1934; 13: 295- 321

9 Yernault JC. The birth of the forced exspiratory manoeuvre: A tribute to Robert Tiffeneau (1910 - 1961). Eur Respir J 1997; 10: 2704 - 2710

10 Bates DV, Christie RV. Respiratory function in disease. 1st ed. Philadelphia and London: WB Saunders, 1964

11 Hogg JC, Macklem T, Thurlbeck WM. Site and nature of airway obstruction in chronic obstructive lung disease. N Engl J Med 1968; 278: $1335-1360$

12 Matthys H. Funktionelle Differentialdiagnostik der Atemwegsobstruktion mittels Ganzkörperplethysmographie. Respiration 1971; 28: 257

13 Ulmer WT, Reichel G, Nolle D. Die Lungenfunktion. Stuttgart: Thieme, 1971

14 Laurell CB, Ericksson S. The electrophoretic alpha-1-globulin pattern of serum in alpha-1-antitrypsin deficiency. Scand J Clin Lab Invest 1963; 15: $132-140$

15 Gross P, Babyak MA, Tolker E, Kaschak M. Enzymatically produced pulmonary emphysema: a prelimjniary report. J Occup Med 1964; 6: $481-484$

16 Sluiter HJ, Koeter GH, de Monchy JG et al. The Dutch Hypothesis (chronic Non-specific Lung disease) revisited. Eur Respir J 1991; 4: 479-489

17 Fletcher C, Peto R, Tinker C, Speizer FE. The Natural History of Chronic Bronchitis and Emphysema. London: Oxford University Press, 1976

18 Anthonisen NR, Connett JE, Kiley JP et al. Effects of smoking intervention and the use of an inhaled anticholinergic bronchodilatator on the rate of decline of FEV1. JAMA 1994; 272: 1497-1505

19 Beske F, Katalinic A, Peters E et al. Morbiditätsprognose 2050. Ausgewählte Krankheiten für Deutschland, Brandenburg und SchleswigHolstein. Schriftenreihe Fritz Beske Institut für Gesundheits-SystemForschung BD 114. Kiel: 2009

20 Vogelmeier C, Buhl C, Criée CP et al. Leitlinie der Deutschen Atemwegsliga und der Deutschen Gesellschaft für Pneumologie und Beatmungsmedizin zur Diagnostik und Therapie von Patienten mit chronisch obstruktiver Bronchitis und Lungenemphysem (COPD). Pneumologie 2007; 61: e1 - e40

21 Decramer M, Celli B, Kesten S et al. Effect of tiotropium on outcomes in patients with moderate chronic obstructive pulmonary disease (UPLIFT): a prespecified subgroup analysis of a randomised controlled trial. Lancet 2009; 374: $1171-1178$

22 Jörres RA, Welte T, Bals $R$ et al. Systemische Manifestationen und Komorbiditäten bei Patienten mit chronisch obstruktiver Lungenerkrankungen (COPD) und ihre Auswirkungen auf den klinischen Zustand und den Verlauf der Erkrankung - eine Übersicht über die Kohortenstudie COSYCONET. DtSCH Med Wochenschr 2010; 135: 446- 499 